having peak densities of more that $10^{15}$ particles $/ \mathrm{cm}^{3}$ and temperatures close to $1 \mathrm{keV}$. The scaling of confinement, proposed on the basis of earlier results in this machine, was confirmed by the Frascati Tokamak which has come into operation during the past year, showing in particular that confinement improves quadratically with the radius of the plasma column. The machine extends the parameter range of plasma currents in high-field machines to $600 \mathrm{kA}$.

The intense work aimed at understanding in more detail, controlling discharges and improving plasma quality has continued. Focusing points here are the magnetohydrodynamic fluctuations, the disruption phenomenon, operation close to the magnetohydrodynamic stability limit, the energy and particle transport mechanisms, and the problems of plasma contamination by impurities and of impurity behaviour in the discharge; arguments where all running tokamaks have contributed new information. Using carbon or steel rather than heavy metal limiters, as well as gettering, has reduced the impurity content of hot low-density discharges appreciably, and the effectiveness of divertors in keeping the plasma clean has been further assessed.

As far as radiofrequency heating is concerned, interesting new results have been reported, in particular for the lower-hybrid frequency range. (EN 9 (1978) $1 / 2$ p. 9). Work done by the JFT-2 group at Tokai and by the PETULA and WEGA teams at Grenoble has shown that the coupling of power to the plasma is good (about 80 to $90 \%$ ) and bacomes even better when the power is increased. At low power levels at least, the heating efficiency is almost as high as for neutral injection (ca. $1 \mathrm{eV}$ ion temperature increase per $\mathrm{kW}$ injected power at plasma densities of about $10^{13}$ particles $/ \mathrm{cm}^{3}$ ) and the plasma confinement is not impaired by the presence of the waves.

The parameter range of Stellerator discharges has been further extended. In Wendelstein VII A at Garching, a density of $10^{14}$ particles $/ \mathrm{cm}^{3}$ has been reached in the discharges. This device as well as $\mathrm{L}-2$ at the Lebedev Institute (Moscow) and CLEO at Culham have further provided new insight into the physics of toroidally confined plasmas.

Work on high- $\beta$ toroidal confinement systems continues on a limited scale. One of the main points here is the investigation of the self-reversal me-

chanism in reversed-field pinches, mainly done in HBTX $I$ at Culham and ETA-BETA I at Padova.

Detailed understanding of the underlying physics of magnetic mirror confinement continues to improve. In 2X II B at Livermore it was shown that plasma confinement definitely becomes better with increasing values of the ratio of plasma radius to the ion Larmor radius. In the attempts to generate close field lines by field reversal, a reduction of the magnetic field on the mirror axis of $90 \%$ has been attained. The tandem mirror concept is intensely studied theoretically and experiments are being prepared. First experimental work on the concept performed on the device Gamma 6 at the University of Tsukuba has been reported and shows clearly an improvement of plasma confinement.

\title{
Seventy-fifth Anniversary of the Spanish Royal Society of Physics and Chemistry
}

On 2 October, the Spanish Royal Society of Physics and Chemistry celebrated its 75 th anniversary. European physics was represented by the President of EPS, Antonino Zichichi who on behalf of the Society conveyed to the President of the Spanish society, Professor J.M. Gamboa Loyarte his warmest greetings. At the commemoration ceremony, held in the presence of is Majesty the King, members of the Government and an international gathering of scientists, Zichichi reminded his audience of the uniqueness of EPS wherein all European countries, regardless of political structure, were represented in one organization that comprised over 3000 Individual Ordinary Members and 28 National Societies and Academies representing some 35000 physicists from all over Europe - East, West, North and South.

The basis for this new unity of geographical Europe, lay in what be called the new culture, viz. the scientific culture. He continued: "As you know, the greatest inventions of human intellect are just three: Language (invented a few hundred thousand years ago), Logic (three thousand years) and Science (just 350 years old). The culture of man today, so-called modern man, is based on and dominated by language. Logic and Science have very little influence on the culture of our time. This is why millions of people can be influenced by just a slogan. A few words, which are an insult to
In the field of inertial confinement a strong effort is made to develop more powerful laser and particle beams for plasma compression. In parallel, the work on the physics of compression and heating of pellets by beams continues.

Conceptual designs of fusion reactors were reported mainly for confinement schemes other than tokamaks, thus filling an important gap, while for tokamaks, attention is being given more and more to economic questions. Furthermore, fusion systems which aim at producing hydrogen gas or at breeding fissile material ("fusionfission hybrid reactors") rather than generating electricity are now under discussion.

F. Engelmann

(Chairman Plasma Physics Division) the human intellect, are enough to create tragedies world wide. The root of the divisions among people of our planet is the fact that logic and science are still not part of modern human culture. As scientists it is our duty to identify all points where unity - not division can be created among people of different religions, classical cultures and political organizations. These points exist in science, and this is why the European Physical Society exists. Your 75 th anniversary coincides with the 10th anniversary of EPS, of which you are an important component. My presence here is proof of how much we want to strengthen the links between the scientists of Spain and the rest of Europe...

It is one of the most important duties of EPS to help all physicists and national societies of Europe to play an active role in the development of our science. It is in this spirit that EPS honours the 75th Anniversary of the Royal Society of Physics and Chemistry of Spain, by awarding to the Society, five scholarships that will allow five Spanish physicists to follow the International Courses at the Ettore Majorana International Centre for Scientific Culture in $1979 . .$.

This month marks ten years of life in the unity of all European physicists and the turning point in a new intensive cultural collaboration among all the physicists of Europe. 